\title{
Hole-transport properties of a furan-containing oligoaryl
}

\author{
Chung-Chih Wu, ${ }^{\text {a) }}$ Wen-Yi Hung, and Tsung-Li Liu \\ Department of Electrical Engineering, Graduate Institute of Electro-Optical Engineering and Graduate \\ Institute of Electronics Engineering, National Taiwan University, Taipei, Taiwan 10617, Republic of China \\ Ling-Zhi Zhang and Tien-Yau Luh \\ Department of Chemistry, National Taiwan University, Taipei, Taiwan 10617 Republic of China \\ and Institute of Chemistry, Academia Sinica, Nankang, Taipei, Taiwan 11529, Republic of China
}

(Received 11 October 2002; accepted 25 February 2003)

\begin{abstract}
We report the carrier transport properties of a furan-containing oligoaryl PF6, which contains no arylamine moiety in the molecular structure but exhibits competitive hole-transport capability in comparison with conventional arylamine-based hole-transport materials often used in organic light-emitting devices (OLEDs) and xerography. Thin films of this compound exhibit both morphological stability and appropriate energy levels for OLED applications. OLEDs using PF6 as the hole-transport layer show low turn-on voltage, high efficiency, and high brightness competitive with those using conventional hole-transport materials, strongly indicating superior hole-transport properties of PF6. The carrier mobility of PF6 was directly measured by the time-of-flight transient photocurrent technique under various temperatures and electric fields. Nondispersive hole transport was observed and a room-temperature hole mobility in excess of $10^{-3} \mathrm{~cm}^{2} / \mathrm{V} \mathrm{s}$ was obtained under high fields. The field and temperature dependence of hole mobility were analyzed and were found consistent with the Bässler (disorder) formalism. (C) 2003 American Institute of Physics.
\end{abstract}

[DOI: $10.1063 / 1.1567056$ ]

\section{INTRODUCTION}

Organic light-emitting diodes (OLEDs) have attracted great interest in recent years because of their applications in efficient, large-area and full-color flat-panel displays. ${ }^{1-4}$ OLEDs in general comprise multiple functional organic layers laminated between a transparent indium-tin-oxide (ITO) anode and a cathode. ${ }^{1-3,5}$ These organic layers provide different functions such as hole transport, electron transport, emission, etc. The virtues of a layered device structure are that it facilitates carrier injection, balances the transport of electrons and holes, and moves the emission region from electrodes to reduce quenching. For the hole-transport layer, arylamine-based derivatives or compounds containing arylamine moieties, which originated from organic photoconductors for xerography, ${ }^{6,7}$ are most widely used due to a few characteristics that are thought essential for an organic compound to be useful as a hole-transport material for OLEDs. ${ }^{1-3,8-11}$ These include the capability in forming morphologically stable amorphous films, superior capability for hole transport, the appropriate ionization potential $\left(I_{p}\right)$ that can match both the work function of the typical ITO anode and the $I_{p}$ of the electron-transport layer or the emitting layer, and the relatively high-lying lowest unoccupied molecular orbital levels to effectively block electrons. Beyond arylamine-based materials, reports of other organic compounds that can meet these requirements as hole-transport materials for OLEDs have been rare and in general in lack of quantitative characterization of hole-transport properties. ${ }^{12,13}$

a) Author to whom correspondence should be addressed; electronic mail: chungwu@cc.ee.ntu.edu.tw
We recently reported that a class of furan-containing oligoaryls that contain no amine moiety could be used as holetransport materials for OLEDs and devices incorporating such compounds exhibited promising characteristics. ${ }^{14,15}$ In the present article, we report the detailed investigation of the carrier-transport properties of such furan-containing oligoaryl by both careful device characterization and by direct measurements of carrier mobility using the time-of-flight transient photocurrent technique.

The article is organized as follows: in Sec. II, experimental methods for material characterization, OLED device fabrication and characterization, and time-of-flight (TOF) mobility measurements are described. In Sec. III, material properties, results and discussions of device characteristics, results and analyses of TOF mobility of the furan-containing oligoaryl are presented in sequence. Finally, Sec. IV concludes results of this work.

\section{EXPERIMENT}

\section{A. Material preparation and characterization}

The furan-containing oligoaryl compound studied in this work is shown in Fig. 1, which is a hexaaryl oligomer containing two phenyl-substituted furan moieties. Accordingly, it was named as PF6, where the number 6 represents the major hexaaryl chromophore, $\mathrm{F}$ the furan moiety, and $\mathrm{P}$ the phenyl substituents on the furan. The synthesis of PF6 was based on a recently reported annulation reaction from the corresponding propargylic dithioacetals, which provides for a convenient synthesis of 2,3,5-trisubstituted furans. ${ }^{14,15}$ Using this protocol, oligoaryls containing symmetrically two furan moieties (e.g., PF6) could be efficiently synthesized through a 


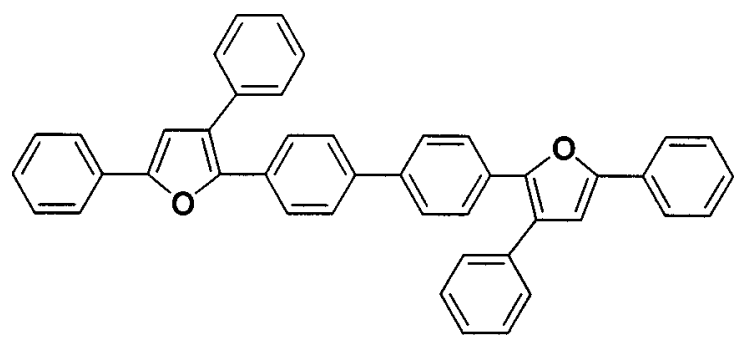

FIG. 1. Molecular structure of PF6.

one-pot synthesis. ${ }^{14,15}$ Synthesized PF6 was subject to purification by temperature-gradient sublimation before use in subsequent analyses. PF6 used in this study was carefully purified twice.

Thermal properties of organic compounds were investigated by thermogravimetric analysis (TGA) and differential scanning calorimetric (DSC). TGA was performed on a TA Instrument 2950 TGA thermal analyzer with a heating rate of $10{ }^{\circ} \mathrm{C} / \mathrm{min}$ under nitrogen purging. DSC was performed on a TA Instrument DSC-2920 low-temperature difference scanning calorimeter. During the DSC measurement, the sample was first heated at a rate of $20^{\circ} \mathrm{C} / \mathrm{min}$ to melt, was quenched with liquid nitrogen, and then was heated again at $10^{\circ} \mathrm{C} / \mathrm{min}$ to record various transition temperatures.

Thin-film samples of organic compounds for optical characterization were prepared by vacuum deposition in a high-vacuum chamber. The UV-visible absorption and photoluminescence (PL) spectra were measured on a Shimadzu UV-1601PC spectrophotometer and on an SLM-AMINCO AB2 luminescence spectrometer, respectively. The PL quantum yield in thin films $\left(\Phi_{\text {film }}\right)$ was determined using a calibrated integrating sphere system. ${ }^{10,16,17}$ The $325 \mathrm{~nm}$ line of the $\mathrm{He}-\mathrm{Cd}$ laser was used to excite the thin-film sample placed in the sphere that was coupled to a cooled chargecoupled-device (CCD) spectragraph and was purged with dry nitrogen. The quantum yield was determined by comparing the spectral intensities of the laser source and the sample emission. In this integrating sphere system, $\Phi_{\text {film }}$ of the typical green OLED material, tris-(8-hydroxyquinolinolato) aluminum $\left(\mathrm{Alq}_{3}\right)$, was determined to be $25 \% \pm 3 \%$, consistent with previously reported values. ${ }^{18,19}$ The ionization potentials $\left(I_{p}\right)$ of organic thin films were measured using atmospheric low-energy photoelectron spectrometer Riken-Keiki $\mathrm{AC}-2 .^{20,21}$

\section{B. Device fabrication and characterization}

To study carrier transport properties of PF6 in organic electroluminescent (EL) devices, OLEDs incorporating PF6 were fabricated and characterized. The devices were fabricated on glass substrates and have the typical structure of multiple organic layers sandwiched between the bottom ITO anode and the top metal cathode. Before use in device fabrication, the ITO-coated glass substrates were carefully cleaned. Cleaned ITO substrates were further treated with UV-ozone right before organic deposition. ${ }^{1}$

The stack of organic layers on ITO in sequence consists of a thin layer of conducting polymer polyethylene dioxythiophene/polystyrene sulphonate (PEDT:PSS) (Bayer Corp.) and other functional layers of small molecular weight. The PEDT:PSS layer served as the hole-injection layer and was deposited by spin coating. ${ }^{22}$ All other material layers in devices, including the metal cathode, were deposited by thermal evaporation in a multiple-source vacuum chamber with a base pressure of $<10^{-6}$ Torr. The deposition rate of organic layers was kept at about $0.2 \mathrm{~nm} / \mathrm{s}$. The deposition system permits the fabrication of the complete device structure in a single vacuum pump-down without breaking vacuum. ${ }^{10}$ The active area of the device is $2 \times 2 \mathrm{~mm}^{2}$, as defined by the shadow mask for cathode deposition.

The current-voltage-brightness $(I-V-L)$ characteristics of EL devices were measured using a source-measurement unit and a $\mathrm{Si}$ photodiode calibrated with Photo $\mathrm{Re}$ search PR-650 spectroradiometer. The EL spectra of OLEDs were taken with a calibrated CCD spectragraph.

\section{Time-of-flight carrier mobility}

To investigate the carrier transport properties in a semiinsulating film, the TOF transient photocurrent technique is often used to directly determine the carrier mobility. ${ }^{23,24}$ In the TOF measurements, the material to be measured is sandwiched between two blocking contacts, with at least one contact to be transparent or semitransparent for illumination. Pulsed illumination through the transparent or semitransparent contact is used to induce photogeneration of a thin sheet of excess carriers near the illuminated contact in the organic layer. Under an applied bias, the photogenerated carriers are swept across the bulk of the organic film toward the other collection electrode, leading to transient photocurrent detected by external circuits. With the same TOF sample configuration, the polarity of the applied bias may be switched to select whether electrons or holes drift across the bulk material and to study independently the transport properties of holes or electrons. The transient photocurrent can be analyzed to yield the transit time $\left(T_{t}\right)$ for carriers to traverse the bulk material. With the applied bias $V$ and the material thickness $D$, the applied electric field $E$ is $V / D$ and the carrier mobility $\mu$ is then given by

$$
\mu=\frac{D}{T_{t} E}=\frac{D^{2}}{V T_{t}} .
$$

In the present work, thick PF6 films $(1.5 \mu \mathrm{m})$ were prepared by vacuum deposition at a rate of $\sim 0.5 \mathrm{~nm} / \mathrm{s}$ onto a glass substrate precoated with semitransparent Ag contact $(30 \mathrm{~nm})$, followed by deposition of the second Al electrode $(150 \mathrm{~nm})$. Ag and Al were chosen as the blocking contacts due to their poor carrier injection capability for PF6.

A frequency-tripled Nd:yttrium-aluminum-garnet laser (355 nm) with $\sim 10$ ns pulse duration was used for pulsed illumination. This wavelength is close to the absorption peak of a PF6 film (377 nm, Fig. 2) and gives a high absorption coefficient of $\sim 10^{5} \mathrm{~cm}^{-1}$, ensuring the penetration depth for the illumination and the sheet of photogenerated carriers to be negligibly thin in comparison with the sample thickness. The transient photocurrent was measured with a series load resistor and was recorded with a digital storage oscilloscope. 


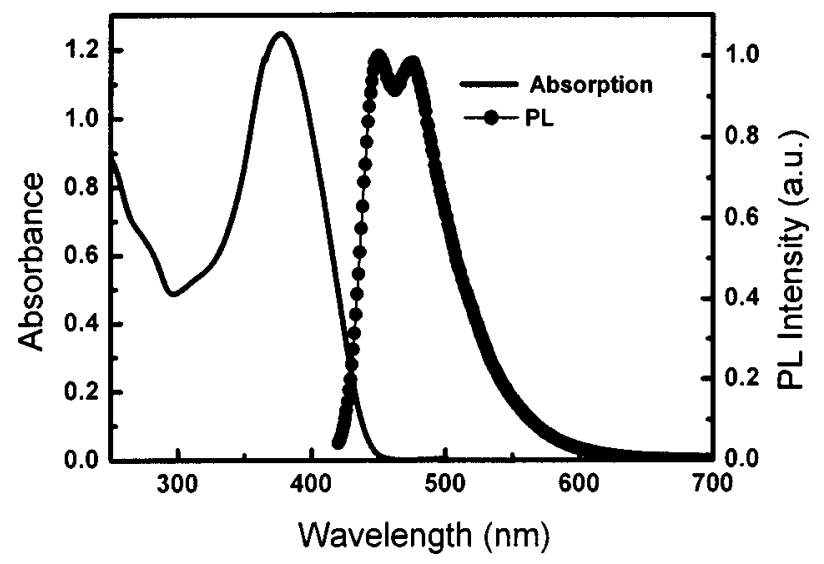

FIG. 2. Absorption and PL spectra of PF6 thin films $(100 \mathrm{~nm})$.

During the measurements, the sample was mounted in the $10^{-5}$ Torr vacuum chamber of a cryostat equipped with a temperature controller, permitting measurements at various temperatures.

Special care had been taken for a few important factors in TOF measurements. First, the intensity of illumination at each measurement condition was kept as low as possible, so that the total charge generated was less than $5 \%$ of the sample capacitor charge to avoid space charge effects that would result in nonuniform distribution of electric field across the sample. Further, the response time of the measurement circuit was always kept substantially smaller than the transit time at a measurement condition.

\section{RESULTS AND DISCUSSIONS}

\section{A. Material properties}

Thermal analyses of PF6 reveal its thermal stability. ${ }^{15}$ In TGA, no weight loss was observed for PF6 up to $320^{\circ} \mathrm{C}$. In DSC, a distinct glass transition temperature $\left(T_{g}\right)$ around $96{ }^{\circ} \mathrm{C}$ and a melting point $\left(T_{m}\right)$ around $235^{\circ} \mathrm{C}$ were observed. The $T_{g}$ of PF6 is similar to that of the popular holetransport material for OLEDs, $\alpha$-naphthylphenylbiphenyl diamine. ${ }^{8}$ As a result, PF6 was able to form homogeneous and stable amorphous films by thermal evaporation. We noticed that PF6 films stayed stable morphologically for months under ambient conditions. Such morphological stability of PF6 may result from the relatively steric and nonplanar structure of the molecule as revealed by x-ray diffraction analysis. ${ }^{14,15}$ In particular, the phenyl substituents at the C-3 positions of furan moieties play important roles in providing steric hindrance to packing and crystallization. ${ }^{15}$

Figure 2 shows the optical spectra of PF6 in thin films. The absorption spectrum of PF6 exhibits an onset at $\sim 450$ $\mathrm{nm}$ and a lowest-energy peak at $377 \mathrm{~nm}$. From the onset of absorption, the optical energy gap of PF6 is estimated to be about $2.76 \mathrm{eV}$. PL of PF6 exhibits blue emission with main peaks around 450 and $475 \mathrm{~nm}$. The PL quantum yield of PF6 in thin films as determined by the integrating sphere system is about $33 \% \pm 3 \%$, which is rather high for a blue emitter in neat films. ${ }^{25,26}$ The steric hindrance inherent with the molecular structure of PF6 presumably accounts for the preservation of the relatively high quantum yield of PF6 in thin

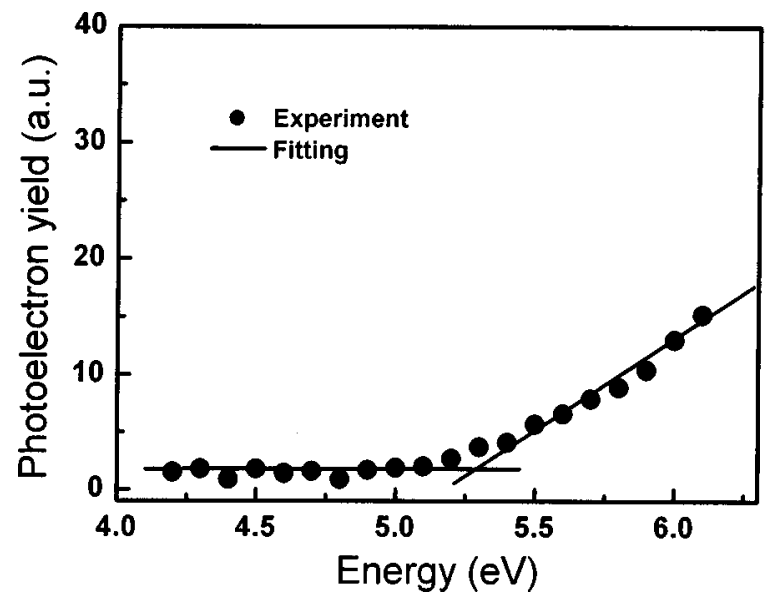

FIG. 3. Photoelectron spectrum of PF6 thin films.

films. Figure 3 shows the photoelectron spectrum of PF6 thin films, which reveals an onset and therefore an ionization potential $\left(I_{p}\right)$ of 5.3-5.4 eV. By subtracting the optical energy gap $(2.76 \mathrm{eV})$ from the measured thin-film ionization potential, one obtains the electron affinity of $\sim 2.6 \mathrm{eV}$ for PF6.

\section{B. EL device characteristics}

Figure 4 shows the $I-V-L$ and efficiency characteristics of an OLED with the configuration of ITO/PEDT:PSS $(\sim 30$ $\mathrm{nm}) / \mathrm{PF} 6(40 \mathrm{~nm}) / \mathrm{Alq}_{3}(60 \mathrm{~nm}) / \mathrm{LiF}(0.5 \mathrm{~nm}) / \mathrm{Al}(150 \mathrm{~nm})$, where the thin $\mathrm{LiF}$ is used as the electron-injection layer. ${ }^{27}$ The EL spectrum of the device, as shown in the inset of Fig. $4(\mathrm{a})$, is characteristic green emission of $\mathrm{Alq}_{3} .{ }^{1}$ The EL properties indicate that in such device configuration, PF6 has functioned as the hole transporter, while $\mathrm{Alq}_{3}$ has functioned as an electron transporter and emitter. In Fig. 4(b), one observes that the device current and emission turn on sharply at a low voltage of $\sim 2 \mathrm{~V}$. Figure 4 (c) shows that the device has a maximum quantum, candela per ampere (cd/A) and power efficiencies of $1.55 \%$ photon/electron, $4.8 \mathrm{~cd} / \mathrm{A}$ and $4 \mathrm{~lm} / \mathrm{W}$, respectively. A further merit of using PF6 as the holetransport layer can be seen in Fig. 4(a), which shows that the device can endure a relatively high current density $(\sim 3000$ $\mathrm{mA} / \mathrm{cm}^{2}$ ) and consequently gives an impressive maximum brightness of nearly $1.3 \times 10^{5} \mathrm{~cd} / \mathrm{m}^{2}$ around $15 \mathrm{~V}$ from nondoped $\mathrm{Alq}_{3}$.

All the earlier device characteristics are among the best of nondoped $\mathrm{Alq}_{3}$-based green OLEDs ever reported, ${ }^{11,27-31}$ which conventionally use arylamine-based hole-transport materials. These results indicate that hole injection and transport characteristics of PF6 are rather good. In terms of hole injection, it is not too surprising since PF6 has an ionization potential (5.3-5.4 eV) similar to arylamine-based materials that have functioned well as hole-transport materials in OLEDs. ${ }^{8-11,32,33}$ Such $I_{p}$ is believed to be a compromise between the work function of the ITO/hole-injection layer $(\sim 4.8-5.2 \mathrm{eV})$ and $I_{p}$ of the electron-transport/emitting layer $\mathrm{Alq}_{3}(\sim 5.6-5.9 \mathrm{eV}) .^{34-40}$ As to the hole-transport capability of PF6, it will be discussed in the following section.

One could also make use of the efficient blue emission of PF6 by having PF6 as an emitting and hole-transport layer 
(a)

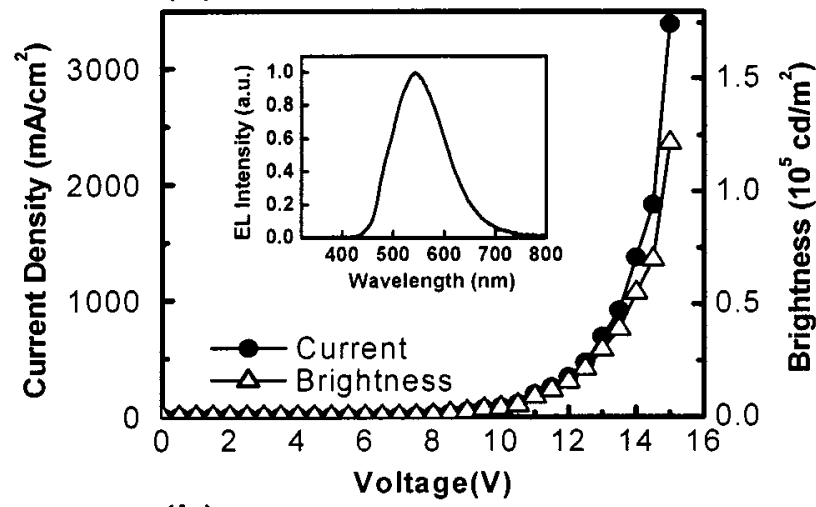

(b)

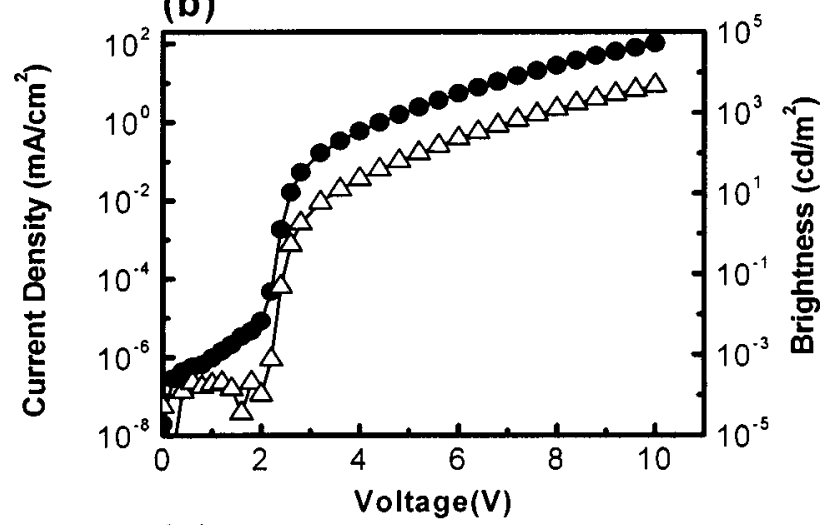

(c)

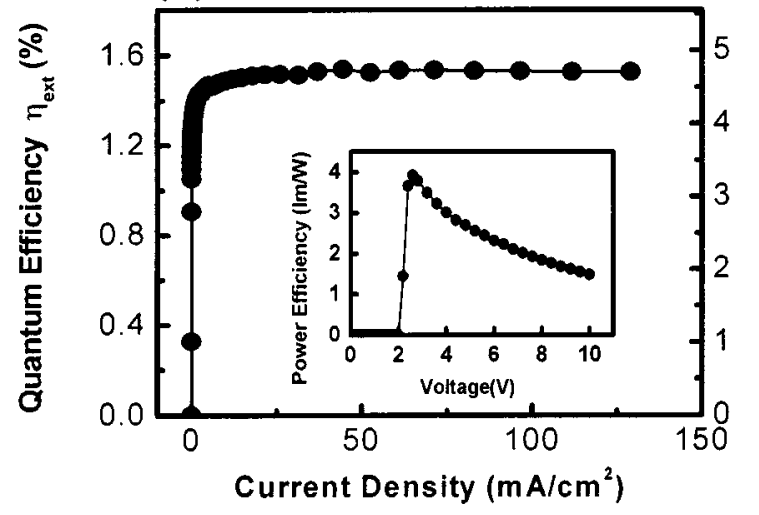

FIG. 4. Device characteristics of ITO/PEDT:PSS $(30 \mathrm{~nm}) / \mathrm{PF} 6(40 \mathrm{~nm}) / \mathrm{Alq} 3$ $(60 \mathrm{~nm}) / \mathrm{LiF}(0.5 \mathrm{~nm}) / \mathrm{Al}(150 \mathrm{~nm})$ : (a) $I-V-L$ characteristics: linear plot, (b) $I-V-L$ characteristics: semilogarithmic plot, and (c) external quantum efficiency and cd/A efficiency vs current. Inset of (a): EL spectrum of the device. Inset of (c): power efficiency vs voltage.

in the device. This can be realized by inserting a holeblocking layer to form a device configuration of ITO/ PEDT:PSS $(30 \mathrm{~nm}) / \mathrm{PF} 6(40 \mathrm{~nm}) / \mathrm{BCP}(10 \mathrm{~nm}) / \mathrm{Alq}_{3}(50$ $\mathrm{nm}) / \mathrm{LiF}(0.5 \mathrm{~nm}) / \mathrm{Al}(150 \mathrm{~nm})$, where BCP represents 2,9dimethyl,4,7-diphenyl,1,10-phenanthroline and has been previously reported as an effective hole-blocking material due to its large $I_{p} \cdot{ }^{41}$ The EL of the device exhibits blue emission with an EL spectrum resembling PL of PF6 [inset of Fig. $5(\mathrm{a})$ ], confirming the effectiveness of BCP in blocking holes and in confining carrier recombination in PF6. Figure 5 shows the $I-V-L$ and efficiency characteristics of such blue OLED. The blue device turns on at a slightly higher voltage of $\sim 2.4 \mathrm{~V}$ and gives a maximum luminance of $\sim 18500$ (a)

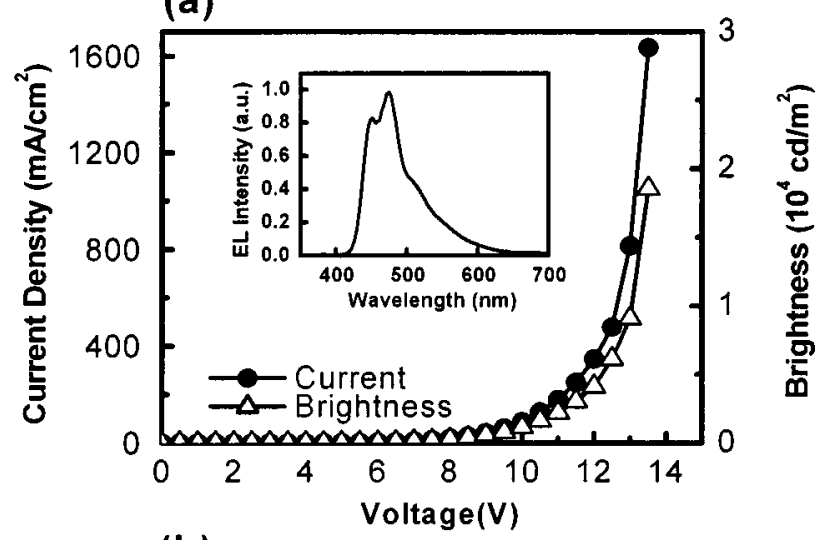

(b)

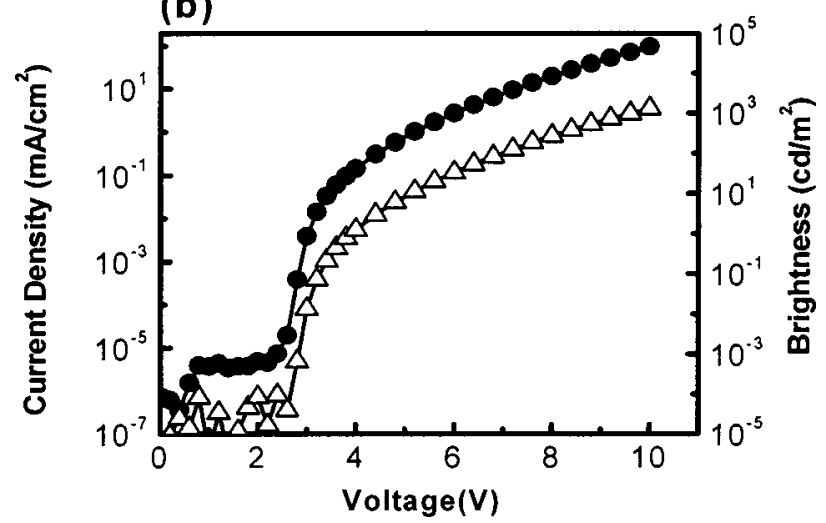

(c)

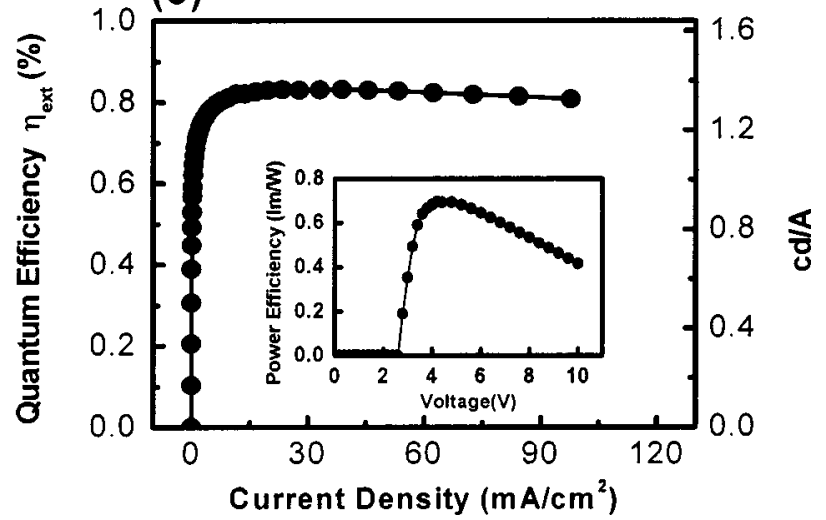

FIG. 5. Device characteristics of ITO/PEDT:PSS $(30 \mathrm{~nm}) / \mathrm{PF} 6(40 \mathrm{~nm}) / \mathrm{BCP}$ $(10 \mathrm{~nm}) / \mathrm{Alq} 3(60 \mathrm{~nm}) / \mathrm{LiF}(0.5 \mathrm{~nm}) / \mathrm{Al}(150 \mathrm{~nm})$ : (a) $I-V-L$ characteristics: linear plot, (b) $I-V-L$ characteristics: semilogarithmic plot, and (c) external quantum efficiency and cd/A efficiency vs current. Inset of (a): EL spectrum of the device. Inset of (c): power efficiency vs voltage.

$\mathrm{cd} / \mathrm{m}^{2}$ at $\sim 13.5 \mathrm{~V}$. The maximum EL quantum, cd/A and power efficiencies are $0.9 \%$ photon/electron, $1.4 \mathrm{~cd} / \mathrm{A}$ and $0.75 \mathrm{~lm} / \mathrm{W}$, respectively.

\section{Charge transport properties}

Results of EL devices strongly suggest that PF6 be rather capable of transporting carriers. To obtain direct measure of the carrier-transport capability of PF6, TOF measurements were carried out on PF6 films. Both electron- and holetransport properties had been examined.

A representative TOF transient for holes is shown in Fig. 6 , which was measured at $300^{\circ} \mathrm{K}$ under an electric field of $2 \times 10^{5} \mathrm{~V} / \mathrm{cm}$ on a $1.5-\mu \mathrm{m}$-thick PF6 film. The short initial 




FIG. 6. Typical TOF transient signal of a $1.5 \mu \mathrm{m}$ PF6 film at $300 \mathrm{~K}$ under an electric field of $2 \times 10^{5} \mathrm{~V} / \mathrm{cm}$. Inset: double logarithmic plot for the same signal.

spike in the transient is limited by the time resolution of the measurement setup. Such initial spike is followed first by a clear constant-current plateau and then by a drop in the current. The final falloff of the current is caused by the holes reaching the collection electrode $(\mathrm{Al})$, where they are discharged. The observation of a current plateau indicates that the charge carriers (i.e., holes) have attained a constant drift velocity and hence nondispersive transport occurs. ${ }^{23}$ The quantum efficiency for photocarrier generation calculated from the total collected charges in the transient photocurrent is $\sim 1.37 \times 10^{-3} .{ }^{42}$ The photogenerated charge is smaller than $5 \%$ of the sample capacitor charge, confirming that the measurement condition was indeed in a regime where the bias field across the sample could be assumed constant. In the double logarithmic representation of the TOF transient (inset of Fig. 6), a carrier transit time, $T_{t}$, can be unambiguously evaluated from the intersection point of the asymptotes to the plateau and the tail sections. In this case, the transit time $T_{t}$ thus determined is $2.23 \times 10^{-6} \mathrm{~s}$, giving a mobility $\mu$ of $3.36 \times 10^{-4} \mathrm{~cm}^{2} / \mathrm{V} \mathrm{s}$ according to Eq. (1). Even higher hole mobility over $10^{-3} \mathrm{~cm}^{2} / \mathrm{Vs}$ was observed at electric



FIG. 7. Hole mobility for PF6 vs square root of the electric field at different temperatures. fields over $5 \times 10^{5} \mathrm{~V} / \mathrm{cm}$ at room temperature (Fig. 7). It should be noted that such mobility value is high and is comparable with the best of amorphous hole-transport materials of arylamine derivatives or any kind. ${ }^{8,23}$

In contrast with the case of holes, the TOF transient for electrons showed rapid decaying signals that no clear transit time could be resolved in both double linear or double logarithmic representations. It is therefore concluded that electron transport of PF6 is, if any, highly dispersive possibly with deep trapping. Thus further discussions of carriertransport properties of PF6 will be focused on those of holes.

Figure 7 shows the field dependence of hole mobilities for PF6 at various temperatures, plotted semilogarithmically as the function of the square root of electric field $\sqrt{E}$. These results can be described as $\mu \propto \exp \left(\beta E^{1 / 2}\right)$, where $\beta$ is a constant that decreases with increasing temperature. Such relationship had been previously observed in different disordered organic systems. ${ }^{8,23,24,29,43-49}$ Early investigations of this nearly universal behavior led to an interpretation in terms of a Poole-Frenkel effect, i.e., field-induced barrier lowering in the course of repetitive detrapping process. ${ }^{50}$ Later studies by Bässler and co-workers, however, revealed that this characteristic dependence could be attributed to the effects of disorder on the hopping conduction in amorphous molecular materials. ${ }^{23,51-55}$

The model developed by Bässler and co-workers, ${ }^{23,51-55}$ commonly referred as the disorder model, is premised on the argument that charge transport occurs by hopping through a manifold of localized states associated with molecules with superimposed energetic and positional disorder. That is, the charge transport can be described as a sequence of fieldassisted one-electron donor-acceptor process. ${ }^{56}$ The model further assumes that the distributions of hopping-site energies and distances are Gaussian. Based on such model and assumptions, Monte Carlo simulation of hopping transport predicts a dependence of the mobility $\mu$ on the electric field and the temperature in the form of Eq. (2), the so-called Bässler formalism

$\mu(E, T)=\mu_{0} \exp \left[-\left(\frac{2 \sigma}{3 k_{B} T}\right)^{2}\right] \exp \left\{C\left[\left(\frac{\sigma}{k_{B} T}\right)^{2}-\Sigma^{2}\right] \sqrt{E}\right\}$.

Here $E$ is the electric field, $T$ is the temperature, $k_{B}$ is Boltzmann's constant, $\mu_{0}$ is a prefactor mobility which represents a hypothetical mobility in the absence of disorder, $\sigma$ is a parameter that characterizes the energy width of the hopping site manifold and therefore the degree of energetic disorder, $\Sigma$ is the parameter to describe the degree of the positional disorder, and $C$ is an empirical constant. The Gaussian disorder model has been quite successful in describing behaviors of carrier transport over a wide range of disordered organic systems. ${ }^{8,23,29,43-49,55}$

By analyzing the mobility data of Fig. 7 in the framework of Bässler formalism, one could extract information regarding $\mu_{0}, \sigma$, and $\Sigma$ and obtain microscopic insight of the PF6 films. Under a zero field, Eq. (2) can be simplified as $\mu(0, T)=\mu_{0} \exp \left[-\left(2 \sigma / 3 k_{B} T\right)^{2}\right]$. Thus, $\mu_{0}$ and $\sigma$ can be de- 


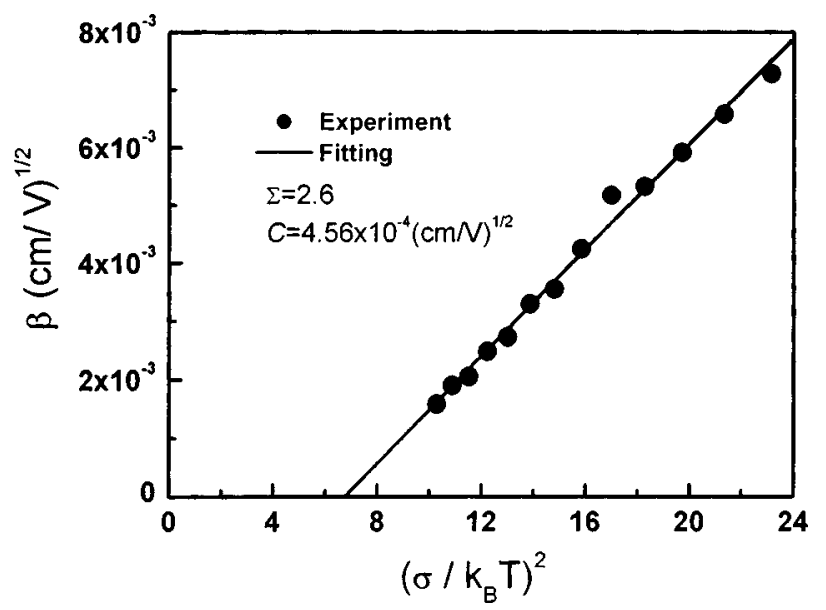

FIG. 8. Zero-field mobility $\mu(0, T)$ for PF6 vs square of the inverse temperature.

termined from the intercept and the slope of the semilogarithmic plot of $\log \mu(E=0)$ vs $T^{-2}$. The zero-field mobilities at various temperatures can be obtained by extrapolating the high-field mobilities in Fig. 7. Figure 8 shows $\log \mu(E=0)$ vs $T^{-2}$ for PF6, which yields $\mu_{0}=0.286 \mathrm{~cm}^{2} / \mathrm{V} \mathrm{s}$ and $\sigma=9.43$ $\times 10^{-2} \mathrm{eV}$.

According to Eq. (2), the slope of the $\log \mu$ vs $E^{1 / 2}$ plot at various temperatures (i.e., $\beta=\partial \ln \left(\mu / \mu_{0}\right) / \partial E^{1 / 2}$ ) is equal to $C\left[\left(\sigma / k_{B} T\right)^{2}-\Sigma^{2}\right]$. From the linear plot of $\beta$ $=\partial \ln \left(\mu / \mu_{0}\right) / \partial E^{1 / 2}$ vs $\left(\sigma / k_{B} T\right)^{2}$, one can determine the empirical constant $C$ from the slope and the positional disorder parameter $\Sigma$ from the intercept $(\beta=0)$, where $\left(\sigma / k_{B} T\right)^{2}$ $=\Sigma^{2}$ holds. Determination of $\sigma$ from the data of Fig. 8 permits such an analysis. $\beta$ values at various temperatures as determined from the data in Fig. 7 are plotted as a function of $\left(\sigma / k_{B} T\right)^{2}$ in Fig. 9. From the results of Fig. 9, $\Sigma$ and $C$ are determined as 2.6 and $4.56 \times 10^{-4}(\mathrm{~cm} / \mathrm{V})^{1 / 2}$, respectively.

The thus extracted $\mu_{0}, \sigma$, and $\Sigma$, and $C$ for PF6 films are found to be within the nominal range of those for various disordered organic systems, in which nondispersive carrier transport had been observed. ${ }^{8,23,29,43-49,55}$ Such consistency suggests that the mechanisms of hole transport in amorphous PF6 films in general be similar to those in most of disordered organic systems.

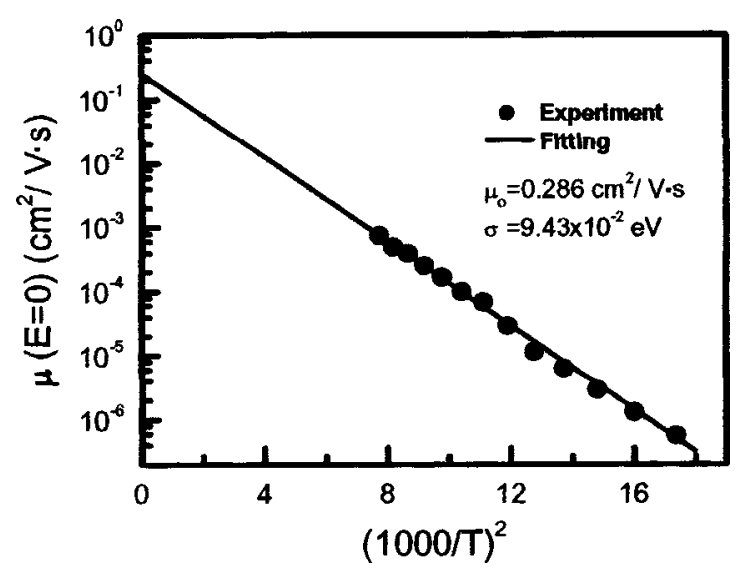

FIG. 9. A plot of $\beta=\partial \ln \left(\mu / \mu_{0}\right) / \partial E^{1 / 2}$ vs $\left(\sigma / k_{B} T\right)^{2}$.

\section{CONCLUSION}

In summary, we report the carrier transport properties of a furan-containing oligoaryl PF6, which contains no arylamine moiety in the molecular structure but exhibits competitive hole-transport capability in comparison with conventional arylamine-based hole-transport materials often used in OLEDs or xerography. OLEDs using PF6 as the holetransport layer give low turn-on voltage, high efficiency, and high brightness competitive with those of OLEDs using conventional hole-transport materials. The carrier-transport mobility of FP6 was studied by the time-of-flight technique under various temperatures and electric fields. Nondispersive hole transport was observed and a room-temperature hole mobility over $10^{-3} \mathrm{~cm}^{2} / \mathrm{V} \mathrm{s}$ was obtained under high fields. The field and temperature dependence of mobility were analyzed and were found consistent with the Bässler (disorder) formalism.

\section{ACKNOWLEDGMENTS}

The authors would like to thank AU Optronics for assistance in $I_{p}$ measurements. This work was supported in part by National Science Council (NSC 91-2215-E-002-029) and Ministry of Education of the Republic of China.

${ }^{1}$ C. W. Tang and S. A. VanSlyke, Appl. Phys. Lett. 51, 913 (1987).

${ }^{2}$ C. W. Tang, S. A. VanSlyke, and C. H. Chen, J. Appl. Phys. 65, 3610 (1989).

${ }^{3}$ C. H. Chen, J. Shi, and C. W. Tang, Macromol. Symp. 125, 1 (1997).

${ }^{4}$ J. H. Burroughes, D. D. C. Bradley, A. R. Brown, R. N. Marks, K. Mackay, R. H. Friend, P. L. Burns, and A. B. Holmes, Nature (London) 347, 539 (1990).

${ }^{5}$ C. Adachi, T. Tsutsui, and S. Saito, Appl. Phys. Lett. 57, 531 (1990).

${ }^{6}$ K.-W. Klupfel, O. Sus, H. Behmenburg, and W. Neugebauer, US Patent No. 3,180,730 (1965).

${ }^{7}$ T. B. Brantly, L. E. Contois, and C. J. Fox, US Patent No. 3,567,450 (1971).

${ }^{8}$ Y. Shirota, J. Mater. Chem. 10, 1 (2000).

${ }^{9}$ D. F. O'Brien, P. E. Burrows, S. R. Forrest, B. E. Koene, D. E. Loy, and M. E. Thompson, Adv. Mater. 10, 1108 (1998).

${ }^{10}$ C. C. Wu et al., Appl. Phys. Lett. 81, 577 (2002).

${ }^{11}$ J. Shi and C. W. Tang, Appl. Phys. Lett. 70, 1665 (1997).

${ }^{12}$ T. Sano, T. Fujii, Y. Nishio, Y. Hamada, K. Shibata, and K. Kuroki, Jpn. J. Appl. Phys., Part 1 34, 3124 (1995).

${ }^{13}$ K. Kawate, Proc. SPIE 2174, 200 (1994).

${ }^{14}$ C.-F. Lee, L.-M. Yang, T.-Y. Hwu, A.-S. Feng, J.-C. Tseng, and T.-Y. Luh, J. Am. Chem. Soc. 122, 4992 (2000).

${ }^{15}$ L.-Z. Zhang, C.-W. Chen, C.-F. Lee, C.-C. Wu, and T.-Y. Luh, Chem. Commun. (Cambridge) 2002, 2336 (2002).

${ }^{16}$ J. C. de Mello, H. F. Wittmann, and R. H. Friend, Adv. Mater. 9, 230 (1997).

${ }^{17}$ K.-T. Wong et al., J. Am. Chem. Soc. 124, 11576 (2002).

${ }^{18}$ H. Mattoussi, H. Murata, C. D. Merritt, Y. Iizumi, J. Kido, and A. H. Kafafi, J. Appl. Phys. 86, 2642 (1999).

${ }^{19}$ D. Z. Garbuzov, S. R. Forrest, A. G. Tsekoun, V. Bulovic, and M. E. Thompson, J. Appl. Phys. 80, 4644 (1996).

${ }^{20}$ H. Kirihata and M. Uda, Rev. Sci. Instrum. 52, 68 (1981).

${ }^{21}$ T. Sano, Y. Hamada, and K. Shibata, IEEE J. Sel. Top. Quantum Electron. 4, 34 (1998)

${ }^{22}$ A. Elschner, F. Bruder, H.-W. Heuer, F. Jonas, A. Karbach, S. Kirchmeyer, S. Thurm, and R. Wehrmann, Synth. Met. 111, 139 (2000).

${ }^{23}$ P. M. Borsenberger and D. S. Weiss, Organic Photoreceptors for Imaging Systems (Marcel Dekker, New York, 1993).

${ }^{24}$ C.-C. Wu, T.-L. Liu, W.-Y. Hung, Y.-T. Lin, K.-T. Wong, R.-T. Chen, Y.-M. Chen, and Y.-Y. Chien, J. Am. Chem. Soc. (in press).

${ }^{25}$ J. Salbeck, N. Yu, J. Bauer, F. Steuber, F. Weissörtel, and H. Bestgen, Synth. Met. 91, 209 (1997). 
${ }^{26}$ M. R. Robinson, S. Wang, G. C. Bazan, and Y. Cao, Adv. Mater. 12, 1701 (2000).

${ }^{27}$ L. S. Hung, C. W. Tang, and M. G. Mason, Appl. Phys. Lett. 70, 152 (1997).

${ }^{28}$ J. Kido, Appl. Phys. Lett. 73, 2866 (1998).

${ }^{29}$ B. J. Chen, W. Y. Lai, Z. Q. Gao, C. S. Lee, S. T. Lee, and W. A. Gambling, Appl. Phys. Lett. 75, 4010 (1999).

${ }^{30}$ T. Wakimoto, Y. Fukuda, K. Nagayama, A. Yokoi, H. Nakada, and M. Tsuchida, IEEE Trans. Electron Devices 44, 1245 (1997).

${ }^{31}$ C.-W. Chen, T.-Y. Cho, C.-C. Wu, H.-L. Yu, and T.-Y. Luh, Appl. Phys. Lett. 81, 1570 (2002).

${ }^{32}$ C. Adachi, K. Nagai, and N. Tamoto, Appl. Phys. Lett. 66, 2679 (1995)

${ }^{33}$ S. Okutsu, T. Onikubo, M. Tamano, and T. Enokida, IEEE Trans. Electron Devices 44, 1302 (1997).

${ }^{34}$ C. C. Wu, C. I. Wu, J. C. Sturm, and A. Kahn, Appl. Phys. Lett. 70, 1348 (1997).

${ }^{35}$ M. G. Mason, L. S. Hung, C. W. Tang, S. T. Lee, K. W. Wong, and M. Wang, J. Appl. Phys. 86, 1688 (1999).

${ }^{36}$ H. Ishii, K. Suguyama, E. Ito, and K. Seki, Adv. Mater. 11, 605 (1999).

${ }^{37}$ S. T. Lee, Y. M. Wang, X. Y. Hou, and C. W. Tang, Appl. Phys. Lett. 74, 670 (1999)

${ }^{38}$ A. Schmidt, M. L. Anderson, and N. R. Armstrong, J. Appl. Phys. 78, 5619 (1995).

${ }^{39}$ A. Rajagopal, C. I. Wu, and A. Kahn, J. Appl. Phys. 83, 2649 (1998).

${ }^{40}$ A. Rajagopal and A. Kahn, J. Appl. Phys. 84, 355 (1998).
${ }^{41}$ Y. Kijima, N. Asai, and S. Tamura, Jpn. J. Appl. Phys., Part 1 38, 5274 (1999).

${ }^{42}$ L.-B. Lin, S. A. Jenekhe, and P. M. Borsenberger, J. Chem. Phys. 105, 8490 (1996).

${ }^{43}$ W. D. Gill, J. Appl. Phys. 43, 5033 (1972).

${ }^{44}$ M. Redecker, D. D. C. Bradley, M. Inbasekaran, W. W. Wu, and E. P. Woo, Adv. Mater. 11, 241 (1999).

${ }^{45}$ P. M. Borsenberger, W. T. Gruenbaum, and E. H. Magin, Jpn. J. Appl. Phys., Part 1 35, 2698 (1996).

${ }^{46}$ L.-B. Lin, S. A. Jenekhe, and P. M. Borsenberger, Appl. Phys. Lett. 69, 3495 (1996).

${ }^{47}$ G. G. Malliaras, Y. Shen, D. H. Dunlap, H. Murata, and Z. H. Kafafi, Appl. Phys. Lett. 79, 2582 (2001).

${ }^{48}$ H. Murata, G. G. Malliaras, M. Uchida, Y. Shen, and Z. H. Kafafi, Chem. Phys. Lett. 339, 161 (2001).

${ }^{49}$ H. Tokuhisa, M. Era, T. Tsutsui, and S. Saito, Appl. Phys. Lett. 66, 3433 (1995).

${ }^{50}$ J. Frenkel, Phys. Rev. 54, 647 (1938).

${ }^{51}$ H. Bässler, Philos. Mag. B 65, 795 (1992).

${ }^{52}$ H. Bässler, Phys. Status Solidi B 15, 175 (1993).

${ }^{53}$ H. Bässler, Int. J. Mod. Phys. 8, 847 (1994).

${ }^{54}$ G. Schönherr, H. Bässler, and M. Silver, Philos. Mag. B 44, 47 (1981).

${ }^{55}$ P. M. Borsenberger, L. Pautmeier, and H. Bässler, J. Chem. Phys. 94, 5447 (1991).

${ }^{56}$ J. S. Facci and M. Stolka, Phys. Rev. B 54, 1 (1986). 